\title{
Correção cirúrgica de drenagem anômala parcial de veias pulmonares direitas em veia cava inferior (síndrome da cimitarra)
}

\author{
Marcos F. BERLINCK*, Januário M. SOUZA*, Salomón O. ROJAS*, Sérgio Almeida de OLIVEIRA*
}

\section{RBCCV 44205-162}

BERLINCK, M. F.; SOUZA, J. M.; ROJAS, S. O.; OLIVEIRA, S. A. - Correção cirúrgica de drenagem anômala parcial de veias pulmonares direitas em veia cava inferior (síndrome da cimitarra). Rev. Bras. Cir. Cardiovasc., 7(1):48-50, 1992.

RESUMO: Paciente adolescente foi operada para correção de drenagem anômala parcial de veias pulmonares direitas em veia cava inferior, com inserção supra-diafragmática. A cirurgia foi realizada com o auxilio da circulação extracorpórea e resultou em ampla comunicação entre a veia anômala e o átrio esquerdo. Os autores discutem as abordagens diagnóstica e cirúrgica desta anomalia.

DESCRITORES: veias pulmonares, drenagem anômala, cirurgia; síndrome da cimitarra.

\section{INTRODUÇĀO}

A síndrome da cimitarra, malformação rara e, às vezes, de difícil diagnóstico ${ }^{1}$ caracteriza-se pela drenagem venosa pulmonar anômala parcial ou total do pulmão direito em veia cava inferior $2,4,7$.

\section{RELATO DO CASO}

Paciente feminina, com 15 anos de idade, apresentando história de cansaço e dispnéia aos grandes esforços desde os 11 anos, que evoluiu para dispnéia aos médios esforços e, aos cinco meses antes da internação, episódios de taquicardia paroxística e cianose perioral.

Ao exame físico, apresentava cianose $+/ 6$, ritmo cardíaco regular, com segunda bulha desdobrada e fixa e sopro sistólico ++, mais audível em borda esternal esquerda. O eletrocardiograma mostrava ritmo sinusal sem sobrecarga de câmaras. A radiografia de tórax mostrou área cardíaca normal e aumento da circulação pulmonar.

Vários estudos ecocardiográficos, inclusive transesofágico, sugeriram diagnósticos diversos (fístula coronário-cavitária e canal arterial persistente), e não foram conclusivos.

Foi realizada planigrafia de tórax, cujo diagnóstico foi de drenagem anômala parcial de veias pulmonares, porém não esclarecendo o local de drenagem. A paciente foi, então, submetida a estudo cineangiocoronariográfico, que mostrou drenagem anômala parcial das veias pulmonares direitas em veia cava inferior, acima dos óstios das veias suprahepáticas. As pressões em tronco pulmonar foram: sistólica $33 \mathrm{mmHg}$, diastólica $13 \mathrm{mmHg}$, média 19 $\mathrm{mmHg}$ e pressão capilar pulmonar $15 \mathrm{mmHg}$. Havia um salto oximétrico de veia cava inferior $(77 \%)$ para o átrio direito $(89 \%)$.

Trabalho realizado no Serviço de Cirúrgia Cardiovascular do Prof. Dr. Sérgio Almeida de Oliveira. Hospital da Beneficência Portuguesa. São Paulo, SP. Brasil.

Recebido para publicação em 11 de março de 1992.

* Do Serviço de Cirúrgia Cardiovascular do Prof. Dr. Sérgio Almeida de Oliveira.

Endereço para separatas: Marcos F. Berlinck. Rua Maestro Cardim, 794. 01323 São Paulo, SP, Brasil. 
BERLINCK, M. F.; SOUZA, J. M.; ROJAS, S. O.; OLIVEIRA, S. A. - Correção cirúrgica de drenagem anômala parcial de veias pulmonares direitas em veia cava inferior (síndrome da cimitarra). Rev. Bras. Cir. Cardiovasc., 7(1):48-50, 1992.

A cirurgia foi realizada com via de acesso através de toracotomia mediana transesternal. A abertura de pleura direita permitiu a visibilização externa da veia anômala, local em que havia aderência que dificultava a abordagem cirúrgica externa. Foi estabelecida circulaçäo extracorpórea com canulação da aorta ascendente e veias cavas superior e inferior e hiportemia sistêmica de $18^{\circ} \mathrm{C}$.

Após atriotomia direita, estendendo-se para a parede anterior de veia cava inferior, identificou-se a drenagem venosa na parade lateral de veia cava inferior, logo acima do óstio das veias supra-hepáticas. O septo interatrial era íntegro.

Para a correção da drenagem anômala, uma comunicação interatrial de aproximadamente $2,5 \mathrm{~cm}$ de diâmetro foi criada com ressecçāo do septo interatrial ao nível da fossa oval. Com um retalho de
PTFEE, fez-se um túnel conectando a veia anômala com o átrio esquerdo, através da nova comunicação interatrial. Um período de parada circulatória total de 50 minutos foi utilizado para facilitar a realização da sutura junto à veia cava inferior. A atriotomia direita foi suturada, incluindo-me um retalho de pericárdio bovino, para ampliar a veia inferior e o átrio direito.

Após aquecimento da paciente para $37^{\circ} \mathrm{C}$, a circulação extracorpórea foi interrompida e realizada a síntese da toracotomia.

A evolução pós-operatória foi boa, sem intercorrências. Antes da alta hospitalar, um novo estudo cineangiográfico foi realizado e mostrava ampla comunicação da veia pulmonar anômala com o átrio esquerdo. A drenagem da veia cava inferior e das veias supra-hepáticas fazia-se normalmente no átrio direito.
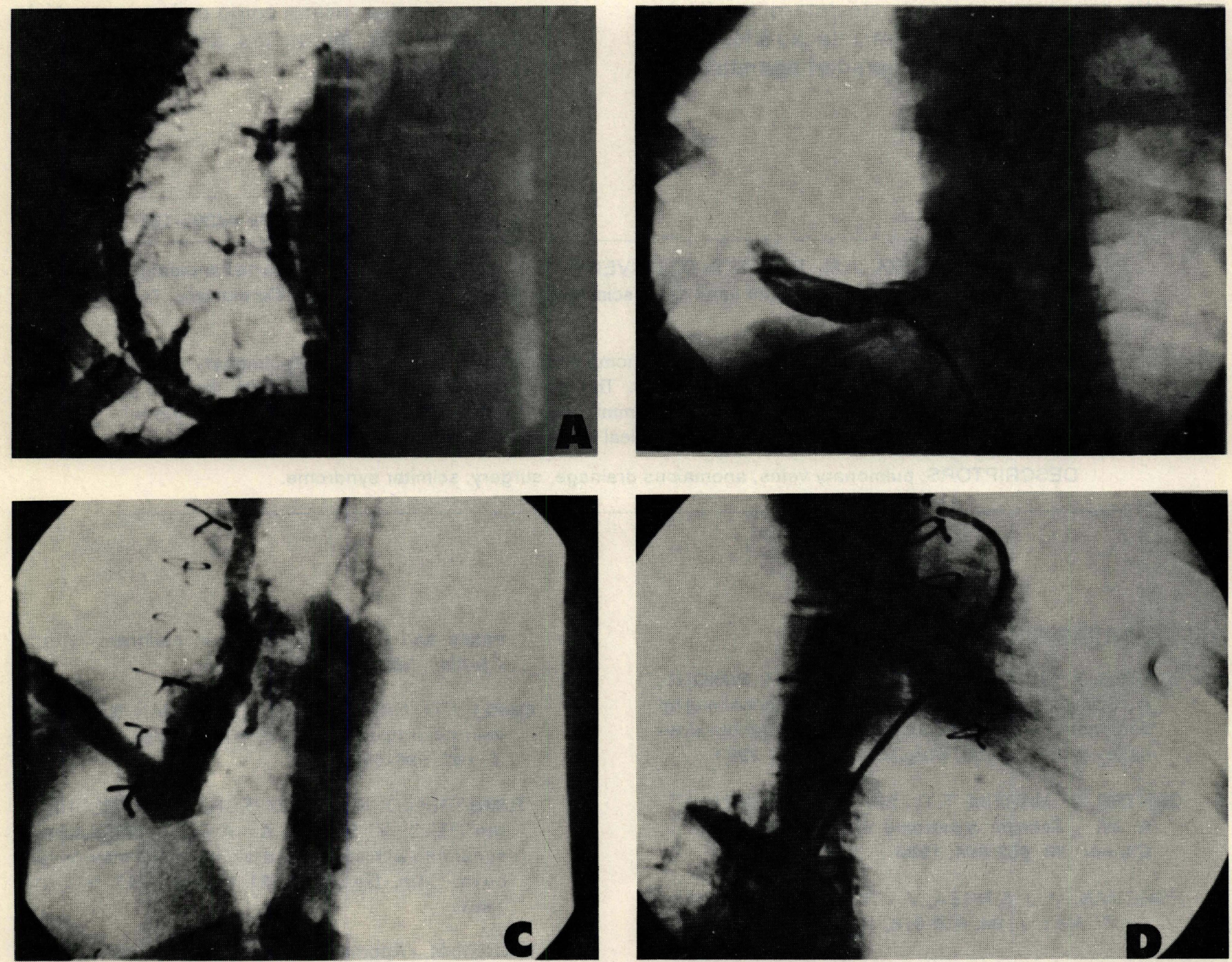

Fig. 1 - Cineangiocardiografias pre e pós-operatórias. Observam-se as veias pulmonares direitas (A) com drenagem em veia cava inferior (B). Na cineangiocardiografia pós-operatória $(\mathrm{C}$ e $\mathrm{D})$ observa-se a drenagem das veias pulmonares direitas no átrio esquerdo através do túnel intraatrial direito. 
BERLINCK, M. F.; SOUZA, J. M.; ROJAS, S. O.; OLIVEIRA, S. A. - Correçāo cirúrgica de drenagem anômala parcial de veias pulmonares direitas em veia cava inferior (síndrome da cimitarra). Rev. Bras. Cir. Cardiovasc., 7(1):48-50, 1992.

\section{COMENTÁRIOS}

A síndrome da cimitarra, neste caso, apresentou, como única alteração anatômica, a drenagem anômala parcial de veias pulmonares direitas em veia cava inferior com inserção supra-diafrag mática. Não estavam presentes outras alteraçōes encontradas nesta síndrome, tais como hipoplasia de ventrículo direito, de artéria pulmonar, ou comunicação interatrial ${ }^{3 \cdot 6}$.

Embora, aos raios $X$ de tórax, houvesse imagem de opacificação no pulmão direito junto à margem da silhueta cardíaca, só se conseguiu o diagnóstico definitivo após realização de planigrafia de tórax, pois os exames ecocardiográficos, inclusive - trans-esofágico, não foram esclarecedores e mostravam diagnósticos totalmente diversos.

O estudo hemodinâmico, além de confirmar o diagnóstico, foi útil no sentido de melhor avaliação da anatomia angiográfica e pressões do sistema arterial pulmonar e, sobretudo, da determinação do local da drenagem anômala com relação à inserção venosa em posição infra ou supradiafragmática, de extrema importância para o planejamento do ato operatório. A indicação cirúrgica, neste caso, deveuse à progressão dos sintomas ${ }^{7}$. A presença de aderências no local de entrada de veia anômala na veia cava inferior, quando inspecionada pela abertura da pleura, impediu que fosse realizada desinserção da veia anômala da veia cava inferior e posterior reimplante no átrio esquerdo.

A técnica operatória utilizada neste caso foi de simples realização. O material utilizado (PTFEE) foi facilmente moldável, resultando em ampla comunicação da veia anômola com o átrio esquerdo.

A utilização de parada circulatória total (PCT) permitiu, com a retirada da cânula da veia cava inferior, que a sutura do patch junto à veia cava fosse realizada sem risco de lesar as veias suprahepáticas.

Devido à rara freqüência com que esta síndrome é encontrada, poucos autores têm larga experiência pessoal e a técnica, assim como o risco operatório, vão depender das alterações anatômicas encontradas em cada caso ${ }^{7}$.

\section{RBCCV 44205-162}

BERLINCK, M. F.; SOUZA, J. M.; ROJAS, S. O.; OLIVEIRA, S. A. - Surgical correction of partial anomalous pulmonary veins drainage in inferior vena cava (scimitar syndrome). Rev. Bras. Cir. Cardiovasc., 7(1): 48-50, 1992.

ABSTRACT: Teenager, presenting congenital anomalous partial drainage of the right pulmonary veins to inferior vena cava in supradiaphragmatic position. The surgical approach was made with the use of extracorporeal circulation and resulted in a wide communication between the anomalous vein and the left atrium. The authors discuss the diagnostic and surgical approach of this rare anomaly.

DESCRIPTORS: pulmonary veins, anomalous drainage, surgery; scimitar syndrome.

\section{REFERÊNCIAS BIBLIOGRÁFICAS}

1 BAXTER, R.; McFADDEN, M.; GRADWAY, M.; WRIGHT, A. - Scimitar syndrome: cinemagnetic resonance imaging demonstration of anoumalous pulmonary venous drainage. Ann. Thorac. Surg., 50: 121-123, 1990.

2

CANTER, C.; MARTIM, T. C.; SPRAY, J. L.; STRAUSS, A. W. - Scimitar syndrome in childhood. Am. J. Cardiol., 58: 652-654, 1986

FOREMAN, M. J. \& ROSA, V. - The scimitar syndrome. South. Med. J., 84: 616-618, 1991.

4 KIELY, B.; FILLER, J.; STUME, S.; DOYLE, E. F. Syndrome of anomalous venous drainage of the right lung to the inferior vena cava: a review of 67 reported cases and three new cases in children. $A m$. J. Cardiol., 20: 102-116, 1967.
5

6
OAKLEY, D.; NAIK, D.; VEREL, D.; ROJAN, S. - Scimitar vein syndrome: report of nine new cases. Am. Heart J., 107: 596-598, 1984.

6 TUMBARELLO, R.; ABBUZZESE, P. A.; MELONI, G.; MARTELLI, V.; SANNA, A. - A variant of the scimitar syndrome with stenosed drainage of the inferior vena cava. Am. Heart J., 121 (2, Parte 2): 616-618, 1991.

7 WONEY, M. - Anomalous pulmonary venous drainage of right lung to inferior vena cava: clinical spectrum in older patients and role of surgery. Q. J. Med. (New series) 44: 463-483, 1977. 